\title{
EDITORIAL
}

\author{
Lei XU, Yanda LI
}

\section{Machine learning and intelligence science: Sino-foreign-interchange workshop on IScIDE2010 (B)}

(c) Higher Education Press and Springer-Verlag Berlin Heidelberg 2011

Aiming at a promotion of Sino-foreign-interchange workshop on Intelligence Science and Intelligent Data Engineering (IScIDE2010), three special issues have been scheduled in Frontiers of Electrical and Electronic Engineering in China. One is called "Emerging Themes on Information Theory and Bayesian Approach" (Vol. 5, No. 3, 2010), which consists of nine invited papers from international well-known pioneers and Chinese senior scientists. The other two special issues are Part A and Part B of the publication of IScIDE2010, a Sino-foreign-interchange workshop on Intelligence Science and Intelligent Data Engineering held on June 3-5, 2010 in Harbin. The Part A came out as Vol. 6, No. 1, 2011 already, including 14 invited presentations by core researchers both from abroad and from Chinese major universities.

The Part B is published here, trisected into three research topics. The first consists of six papers on learning algorithms and model selection. The second contains four papers that relate to sequence analysis and temporal modeling from different fields of applications, including radar HRRP recognition, RNA sequence analysis, speech processing, and control systems. The third one is the largest part that consists of nine papers on image analysis and machine vision, covering natural scene recognition, outdoor video analysis, MRI image segmentation, biometrics recognition, quality inspection, color vision, forum data analysis, etc.

Among the total of 19 papers, the two-third comes from major universities in mainland of China while the rest comes from universities in Hong Kong (China), England, and Finland. Except the last four papers that are contributed by young researchers as first authors, the first authors of all the other papers are Ph.D candidates. Actually, this special issue provides a typical perspective for international communities to observe the researches of Ph.D candidates in China, though it may not completely represent a list of all the topmost ones, as students at some universities are required to publish their papers on those so-called "SCI journals".

Finally, we would like to express our gratitude to all the authors on their valuable supports to this special issue.

Acknowledgements This work by the guest editor was partially supported by the National Key Basic Research Development Program of China (No. 2009CB825404). The guest editor is a member of the Steering Committee for Information Sciences, to which this work was partially dedicated.

Lei XU

Guest Editor of the Special Issue

Yanda LI

Editor-in-Chief of Frontiers of Electrical and Electronic Engineering in China

Received May 6, 2011

Lei XU

Department of Computer Science and Engineering, The Chinese University of Hong Kong, Hong Kong, China

E-mail: lxu@cse.cuhk.edu.hk

Yanda LI

Department of Automation, Tsinghua University, Beijing 100084, China

E-mail: daulyd@tsinghua.edu.cn 